\title{
Identifying genetic variation affecting a complex trait in simulated data: a comparison of meta-analysis with pooled data analysis Xiaodong $\mathrm{Wu}^{*}$, Donghui Kan, Richard S Cooper and Xiaofeng Zhu
}

Address: Department of Preventive Medicine and Epidemiology, Loyola University Chicago Medical Center, Maywood, IL, USA

Email: Xiaodong Wu* xwu@apache.medctr.luhs.org; Donghui Kan - dkan@lumc.edu; Richard S Cooper - rcooper@lumc.edu; Xiaofeng Zhu -xzhu1@lumc.edu

* Corresponding author

from Genetic Analysis Workshop 14: Microsatellite and single-nucleotide polymorphism

Noordwijkerhout, The Netherlands, 7-10 September 2004

Published: 30 December 2005

BMC Genetics 2005, 6(Suppl I):S97 doi:I0.1/86/147|-2156-6-SI-S97

\begin{abstract}
We explored the power and consistency to detect linkage and association with meta-analysis and pooled data analysis using Genetic Analysis Workshop 14 simulated data. The first 10 replicates from Aipotu population were used. Significant linkage and association was found at all 4 regions containing the major loci for Kofendrerd Personality Disorder (KPD) using both combined analyses although no significant linkage and association was found at all these regions in a single replicate. The linkage results from both analyses are consistent in terms of the significance level of linkage test and the estimate of locus location. After correction for multiple-testing, significant associations were detected for the same 8 single-nucleotide polymorphisms (SNP) in both analyses. There were another 2 SNPs for which significant associations with KPD were found only by pooled data analysis. Our study showed that, under homogeneous condition, the results from meta-analysis and pooled data analysis are similar in both linkage and association studies and the loss of power is limited using meta-analysis. Thus, meta-analysis can provide an overall evaluation of linkage and association when the original raw data is not available for combining.
\end{abstract}

\section{Background}

Identifying the susceptibility genes for human complex traits, such as obesity, diabetes, and hypertension, represents a challenging task for human geneticists. Due to the moderate effect of each gene on the trait, it is difficult to acquire enough power to detect all disease susceptibility genes in one study with a moderate sample size. One potential solution to this challenge is to combine the primary studies to increase the power to identify the genes with small effects. When the raw data is available, pooling raw data should be the most powerful method to combine studies. However, in practice, raw data is usually hard to obtain and the alternative is to pool the results (metaanalysis) of the primary studies instead.
Although meta-analysis has been widely used in clinical trials and epidemiological studies, it is a relatively new approach in linkage studies. Methods for meta-analysis of linkage analysis can be roughly divided into two categories. In the first category, individual effect size and its variance are available for each study and can be combined using a fixed-effects or random-effects model to obtain an overall effect. Li and Rao [1] proposed to use regressioncoefficient estimates from the Haseman-Elston (HE) regression [2] as effect size and combine them using a random-effects model in sib-pair analysis. Gu et al. [3] developed a similar approach. They used the proportion of alleles shared identical by descent (IBD) among selected sib pairs as their effect size. The advantage of using such methods is that heterogeneity among studies can be estimated and tested, and an overall effect size then esti- 
mated. The second category includes methods for combining significance levels, such as $p$-values or LOD scores. The advantage of such methods is that it does not require a common effect size, only that every study tests the same null hypothesis. Thus, these methods are quite general and flexible to use. The very first method for combining results from different studies to obtain consensus was developed by R. A. Fisher in 1925 [4]. Fisher's method of combining $p$-values is remarkably general and easy to use. Wise et al. [5] developed a nonparametric meta-analysis method for genome scans (GSMA) that is based on either $p$-value or LOD score ranks. In this method, each chromosome is separated into independent bins with equal spacing, and the linkage evidence for each bin is ranked in each study. To combine the linkage evidence from different studies, an average rank is calculated for each bin across the studies.

The main aim for this analysis is to identify the underlying genetic factors responsible for Kofendrerd Personality Disorder (KPD) using pooled data analysis and meta-analysis and to further compare the power to detect linkage and association with these two methods.

\section{Methods \\ Data selection}

The analysis was performed in the Aipotu population. The diagnosis for KPD in this population is based on all 3 different criteria, which is the most heterogeneous compared with the other 3 populations and represents the most realistic case. Replicates 1-10 were selected for the analysis, which represents a reasonable sample size for the metaanalysis of genome scans in reality.

\section{Combined linkage analysis}

Fisher's method of combining $p$-values was used in our analysis considering that 1 ) in practice, the designs and applied statistical methods are usually different in linkage analyses and it is difficult to obtain a common effect size for combining; 2) we can combine significance level at every marker location using Fisher's method, but only at every bin $(\sim 30 \mathrm{cM})$ using GSMA.

Linkage analysis with microsatellite markers was performed using a nonparametric allele-sharing method [6] implemented in software ALLEGRO [7] in each of the 10 replicates and also in the pooled data. This method evaluates linkage by testing excessive IBD sharing within affected relatives. We chose $Z_{l r}[6]$ as the test statistic for linkage. Under the null hypothesis of no linkage, $Z_{l r}$ asymptotically follows a normal distribution. We then combined the results of the 10 primary genome scans using Fisher's method of combining $p$-values [4]. This method is based on the observation that if $n$ independent tests are made of the same hypothesis, then we can calculate a combined $p$-value for all $n$ tests by $\sum_{i=1}^{n}\left(-2 \ln p_{i}\right)$, where $p_{i}$ is the significance level for study $i$. The combined $p$-value is asymptotically distributed as a chi-square distribution with $2 x n$ degrees of freedom. Under the null hypothesis of no linkage, $4.6 \times$ LOD follows a chi-square distribution with 1 degree of freedom. In practice, the test is one-sided because it is only declared significant when $\hat{\theta}$ $<1 / 2$, where $\hat{\theta}$ is the estimated recombination rate. The combined $p$-value was transformed to LOD scores by calculating the quantile of the chi-square distribution.

\section{Combined association analysis}

Hardy-Weinberg equilibrium (HWE) was tested in founders using software package ARLEQUIN [8]. Pair-wise linkage disequilibrium (LD) was calculated as $\mathrm{D}^{\prime}$ and $\mathrm{r}^{2}$ [9] using ldmax routine in software GOLD http:// www.sph.umich.edu/csg/abecasis/GOLD in each replicate as well as in pooled data. The $p$-values were corrected for multiple testing for the number of single-nucleotide polymorphisms (SNPs) tested for association. To account for the non-independence between these SNPs, a spectral decomposition method was used to obtain the effective number of independent SNPs [10]. This method has been implemented in the software SNPSpD http:// genepi.qimr.edu.au/general/daleN/SNPSpD/. SNPs with allele frequency less than $5 \%$ were excluded from the analysis.

To further narrow down the regions containing the genetic variants for KPD, we applied family association test implemented in software package FBAT (version 1.5.1) [11] for all of the SNPs within the 1-LOD intervals of linkage peaks from fine mapping study. FBAT builds on the original TDT method [12] and in particular puts tests of different genetic models, tests of different sampling designs, tests involving different disease phenotypes, tests with missing parents, and tests of different null hypothesis all in the same framework. Because our association tests were performed in the regions showing linkage evidence, our null hypothesis is linkage but no association. To account for the correlation among relatives caused by linkage, we used the empirical variance [13] in calculating test statistic. The results of FBAT analyses at each SNP were combined using Fisher's method as described above.

\section{Results}

Four regions were found showing significant evidence for linkage (LOD > 3.6 [14]) in the meta-analysis of initial genome scans. The markers with highest LOD score at these regions are: D01S0023 (LOD = 7.7) on chromo- 
Table I: Fine mapping results from meta-analysis and pooling raw data

\begin{tabular}{ccccc}
\hline & & \multicolumn{3}{c}{ LOD } \\
\cline { 3 - 4 } Chromosome & Marker & Meta-analysis & Pooled raw data & I-LOD Region \\
\hline 1 & COIR0052 & 11.6 & 13.2 & D0IS0023-C0IR0053 \\
3 & C03R028I & 19.0 & 20.9 & C03R0279-C03R028I \\
5 & C05R0380 & 14.6 & 16.9 & C05R0379-C05R038I \\
9 & C09R0765 & 14.3 & 15.0 & C09R0764-C09R0766 \\
\hline
\end{tabular}

some 1, D03S0127 (LOD = 19.0) on chromosome 3, D05S0173 (LOD = 13.9) on chromosome 5, and D09S0348 $($ LOD $=11.2)$ on chromosome 9. Significant evidence for linkage was also found with the same markers in the pooled data analysis: D01S0023 (LOD = 9.8), D03S0127 (LOD = 20.2), D05S0173 (LOD = 15.6), and D09S0348 (LOD = 11.6).

Table 2: Result of family-based association test using FBAT

\begin{tabular}{|c|c|c|}
\hline SNPa & p-meta & $p$-all \\
\hline \multicolumn{3}{|l|}{ Chromosome I } \\
\hline B0IT0545 & 0.0138 & 0.1447 \\
\hline В0IT0549 & 0.0341 & 0.0293 \\
\hline B0IT055I & 0.1946 & 0.0398 \\
\hline В0IT0558 & 0.3482 & 0.0487 \\
\hline COIR0052 & 0.0316 & 0.3425 \\
\hline \multicolumn{3}{|c|}{ Chromosome 3} \\
\hline В03Т3052 & 0.0329 & 0.0043 \\
\hline В03T3056 & $<0.0001$ & $<0.0001$ \\
\hline В03T3057 & $<0.0001$ & $<0.0001$ \\
\hline В03T3058 & $<0.0001$ & $<0.0001$ \\
\hline C03R028I & $<0.0001$ & $<0.0001$ \\
\hline В03Т3066 & 0.0584 & 0.0021 \\
\hline \multicolumn{3}{|c|}{ Chromosome 5} \\
\hline B05T4I36 & 0.0006 & $<0.0001$ \\
\hline B05T4|43 & 0.0021 & 0.0171 \\
\hline B05T4I45 & 0.5868 & 0.0409 \\
\hline B05T4I46 & 0.0016 & 0.0065 \\
\hline \multicolumn{3}{|l|}{ Chromosome 9} \\
\hline В09T833 I & $<0.0001$ & $<0.0001$ \\
\hline В09T8333 & $<0.0001$ & $<0.0001$ \\
\hline C09R0765 & 0.0449 & 0.0004 \\
\hline В09Т8337 & 0.1199 & 0.0010 \\
\hline В09Т8338 & 0.5018 & 0.0439 \\
\hline В09T8340 & $<0.0001$ & $<0.0001$ \\
\hline В09Т834I & 0.0074 & $<0.0001$ \\
\hline C09R0766 & 0.4118 & 0.0373 \\
\hline
\end{tabular}

aSNPs are highlighted in bold if the $p$-values are less than 0.0006 in both combined analyses.
Fine mapping of KPD by linkage analysis was further performed at these regions by analyzing additional SNP markers. The results from meta-analysis and pooled data analysis are presented in Table 1. To further narrow down the regions containing the disease loci, SNPs within 1LOD intervals were tested for association using FBAT. A total of $17,26,20$, and 20 SNPs were tested at linkage regions on chromosomes $1,3,5$, and 9. In the HWE tests, only one SNP, B01T0564, significantly deviated from the equilibrium $(p=0.0001)$ after adjustment for multiple testing in pooled data. LD was calculated around the linkage regions on chromosomes 1, 3, 5, and 9. The LD measurements from individual replicate and pooled data are similar. We defined strong LD as $\mathrm{D}^{\prime}>0.90$ or $\mathrm{r}^{2}>0.30$ [15]. No strong LD was found at the linkage region on chromosome 1 (maximum $\mathrm{D}^{\prime}=0.683$ and maximum $\mathrm{r}^{2}=$ 0.024). Strong LD was found around B03T3063B03T3065 region on chromosome 3, B05T4141-B054143 region on chromosome 5, and B09T8337-B09T8339 region on chromosome 9.

The FBAT results for markers with nominal significance ( $p$ $<0.05$ ) in either meta-analysis or pooled data analysis are presented in Table 2. Analyses using SNPSpD showed that the overall correlations across all SNPs in each region are weak and could be ignored. Thus multiple testing was adjusted using Bonferroni correction, which gave $\alpha=$ $0.05 / 83=0.0006$ as the adjusted significance level. Significant association was detected for 8 SNPs (B03T3056, B03T3037, В03T3058, C03R0281 on chromosome 3; B05T4136 on chromosome 5; B09T8331, B09T8333 and B09T8340 on chromosome 9) in both analyses based on $\alpha=0.0006$. Two SNPs on chromosome 9 achieved significance only in pooled data analysis. They are C09R0765 ( $p$ $=0.0004)$ and B09T8341 $(p<0.0001)$.

No significant association was detected based on $\alpha=$ 0.0006 for chromosome 1 in either analysis. This could be caused by the small genetic effect, low frequency of causal disease mutation, or low LD between causal SNP with other SNP markers. 


\section{Discussion}

All 4 regions containing the major loci were correctly identified in the 2 combined linkage analyses, although no single replicate showed significant linkage evidence at all the regions. However, no significant linkage was found for the 2 modified loci on chromosome 2 and 10 even with combined analyses. This is most likely caused by the small effects of these 2 loci on the disease phenotypes. The linkage results are consistent for both analyses with the similar 1-LOD interval and significance level. However, the maximum LOD scores at the linkage regions are consistently lower in the meta-analysis than those in the pooled raw data analysis, which means that we may lose some power to detect linkage using meta-analysis although the loss is limited in our case.

We concentrated on single-SNP analysis in our association studies. The results of association analyses are quite consistent in both combined analyses. Significant association $(p<0.0006)$ was detected in both analyses for 8 out of 10 SNPs showing significant association in either analysis (Table 2). Two SNPs achieved significance only in pooled data analysis, which suggests that the power to detect association may be also higher in pooled data analysis.

An important assumption of meta-analysis using Fisher's method of combining $p$-values is that the primary studies are independent from each other. Bias could be caused by including studies with overlapping samples in the metaanalysis. This could be avoided by a careful check of authorship of the publications and detailed information about these studies. However, this may not be possible to do under some circumstances. If a serious overlap is found, an easy solution to prevent such bias is to leave some studies out. However, we may waste useful information by doing this. New statistical methods will be needed to incorporate such dependency in the meta-analysis.

The 10 replicates we chose for this analysis differ only in sampling and no heterogeneity exists among them. Our results show that meta-analysis has a similar power to detect linkage and association compared with pooled data analysis under homogeneous condition. In reality, different studies could differ in study design, marker set, statistical analysis and etc., further investigation need to be done to evaluate the effect of these factors on meta-analysis.

\section{Conclusion}

Our analysis showed that, under homogeneous condition, the results from meta-analysis with Fisher's method and pooled data analysis are similar and the loss of power to detect linkage and association is limited for meta-analysis. Thus, meta-analysis can provide an overall evalua- tion of linkage and association when the original raw data is not available under this condition. More studies need to be done to investigate the power of meta-analysis when heterogeneity exists among primary studies.

\section{Abbreviations}

GSMA: Genome-scan meta-analysis

\section{HE: Haseman-Elston}

HWE: Hardy-Weinberg equilibrium

IBD: Identical by descent

KPD: Kofendrerd Personality Disorder

LD: Linkage disequilibrium

SNP: Single-nucleotide polymorphism

\section{Acknowledgements}

This study was supported in part by grant HL74I66 from National Heart, Lung, and Blood Institute.

\section{References}

I. Li Z, Rao DC: Random effects model for meta-analysis of multiple quantitative sibpair linkage studies. Genet Epidemiol 1996, 1 3:377-383.

2. Haseman JK, Elston RC: The investigation of linkage between a quantitative trait and a marker locus. Behav Genet 1972, 2:3-19.

3. Gu C, Province M, Todorov A, Rao DC: Meta-analysis methodology for combining non-parametric sibpair linkage results: genetic homogeneity and identical markers. Genet Epidemiol 1998, 15:609-626.

4. Fisher RA: Statistical Methods for Research Workers London: Oliver and Boyd, Ltd; 1925:99-I0I.

5. Wise LH, Lanchbury JS, Lewis CM: Meta-analysis of genome searches. Ann Hum Genet 1999, 63:263-272.

6. Kong A, Cox NJ: Allele-sharing models: LOD scores and accurate linkage tests. Am J Hum Genet 1997, 61:1 I79-1 I 88.

7. Gudbjartsson DF, Jonasson K, Frigge ML, Kong A: Allegro, a new computer program for multipoint linkage analysis. Nat Genet 2000, 25:12-13.

8. Schneider S, Roessli D, Excoffier L: ARLEOUIN version 2.000: an exploratory population genetics software environment. Geneva, Switzerland:Genetics and Biometry Laboratory, University of Geneva; 1997.

9. Delvin B, Risch N: A comparison of linkage disequilibrium measures for fine-scale mapping. Genomics 1995, 29:3 I I-322.

10. Nyholt DR: A simple correction for multiple testing for singlenucleotide polymorphisms in linkage disequilibrium with each other. Am J Hum Genet 2004, 74:765-769.

II. Rabinowitz D, Laird N: A unified approach to adjusting association tests for population admixture with arbitrary pedigree structure and arbitrary missing marker information. Hum Hered 2000, 50:21I-223.

12. Spielman RS, McGinnis RE, Ewens WJ: Transmission test for linkage disequilibrium: the insulin gene region and insulindependent diabetes mellitus (IDDM). Am J Hum Genet 1993, 52:506-516.

13. Lake SL, Blacker D, Laird NM: Family-based tests of association in the presence of linkage. Am J Hum Genet 2000, 67:1515-1525.

14. Lander E, Kruglyak L: Genetic dissection of complex traits: guidelines for interpreting and reporting linkage results. Nat Genet 1995, II:24I-247.

15. Ardlie KG, Kruglyak L, Seielstad M: Patterns of linkage disequilibrium in the human genome. Nat Rev Genet 2002, 3:299-309. 